•综述・

\title{
生物间高阶相互作用研究进展
}

\author{
李远智 肖俊丽 刘翰伦 王酉石 储诚进 ${ }^{*}$ \\ (中山大学有害生物控制与资源利用国家重点实验室, 中山大学生命科学学院, 广州 510275)
}

\begin{abstract}
摘要: 生物间的相互作用是物种共存和生物多样性维持的关键。传统的物种共存研究主要关注配对物种之间的直 接相互作用, 而忽略了更为复杂的间接相互作用。本文首先介绍了两种间接相互作用: 链式相互作用(本质上仍是 两两物种之间的相互作用)和高阶相互作用。在此基础上, 我们回顾了高阶相互作用定义的演变历史(包括狭义的 高阶相互作用和广义的高阶相互作用)及其检验方法, 并介绍了高阶相互作用在多营养级之间和同一营养级内的 研究概况。目前, 生态学家主要对多营养级之间(如食物网)的高阶相互作用的特征、发生机制、作用途径及实验 证据等方面进行了详尽的研究。近年来, 同一营养级内的高阶相互作用也开始受到关注, 因此我们进一步介绍了 同一营养级内个体水平高阶相互作用的重要意义和度量方法。从个体水平上研究高阶相互作用, 既能统一狭义和 广义高阶相互作用在定义上的争议, 又可以将个体间的差异(如个体大小、个体的空间分布等信息)考虑进来。最 后, 本文对高阶相互作用一些可能的重要研究方向进行了展望: 在自然群落中(尤其同一营养级内)检验高阶相互 作用的普遍性与相对重要性, 探讨高阶相互作用的发生机制以及如何将高阶相互作用整合到现有的理论体系中 等。高阶相互作用的研究有助于我们全面深刻地理解物种共存和生物多样性的维持机制, 丰富和完善群落生态学 的理论框架, 为人类世背景下的生物多样性保护和生态系统功能维持与提升提供基础。
\end{abstract}

关键词: 密度介导的间接相互作用; 性状介导的间接相互作用; 相互作用的调节; 个体水平高阶相互作用; 非线 性密度制约; 营养级; 生态网络

\section{Advances in higher-order interactions between organisms}

\author{
Yuanzhi Li, Junli Xiao, Hanlun Liu, Youshi Wang, Chengjin Chu* \\ State Key Laboratory of Biocontrol, School of Life Sciences, Sun Yat-sen University, Guangzhou 510275
}

\begin{abstract}
It is well known that interactions between organisms are the key to species coexistence and biodiversity maintenance. Traditional studies focused overwhelmingly on direct interactions between species pairs, ignoring the more complex indirect interactions. In this review, we first distinguished two types of indirect interactions, i.e. interaction chains and higher-order interactions (HOIs). Then we reviewed the definition of higher-order interactions including the hard-HOIs and soft-HOIs, and the studies of HOIs among multiple trophic levels and within a single trophic level. In the food-web literature (among multiple trophic levels), ecologists widely studied the properties, mechanisms, pathways and experimental evidence of HOIs. Recently, there is an increasing interest in HOIs within a single trophic level. Therefore, we further introduced the significance and quantification of individual-level HOIs within a single trophic level. Not only can individual-level HOIs reconcile the hard-HOIs and soft-HOIs, but also allow us to consider variatons between individuals (e.g. individual size and spatial distribution). Finally, we proposed some promising research directions of HOIs including but not limited to: testing the prevalence and relative importance of HOIs in natural communities, exploring the mechanisms of HOIs and integrating HOIs to existing theories of community ecology. Inclusion of HOIs will help us understand the mechanisms of species coexistence and biodiversity maintenance profoundly and comprehensively, enrich and refine the theoretical framework of community ecology, and lay the foundation for biodiversity conservation and management of ecosystems in the Anthropocene.
\end{abstract}

收稿日期: 2020-08-14; 接受日期: 2020-09-15

基金项目: 国家自然科学基金(31925027; 31622014; 31570426; 31901106)和中国博士后科学基金(2018M643295)

* 通讯作者 Author for correspondence. E-mail: chuchjin@mail.sysu.edu.cn 
Key words: density-mediated indirect interactions; trait-mediated indirect interactions; interaction modifications; individual-level higher-order interactions; nonlinear density dependence; trophic levels; ecological network

多物种共存机制是群落生态学的核心研究内 容, 是生物多样性保护的基础, 其关键是生物间的 相互作用。从19世纪的Logistic种群增长模型(种内 直接相互作用, Verhuls, 1838), 到 20 世纪的 Lotka-Volterra竞争模型(种内和种间的直接相互作 用, Lotka, 1925; Volterra, 1926), 再到21世纪的当代 物种共存理论(生态位差异与适合度差异, Chesson, 2000, 2018; 储诚进等, 2017)的近200年研究中, 生 物间的直接相互作用一直是人们理解自然系统的 关键机理和主体思想。除了成对物种之间的直接相 互作用, 如竞争、互惠(如传粉、植物种子的动物传 播等行为)、促进、(拟)寄生、捕食等, 人们很早就 意识到多物种之间的间接相互作用(Darwin, 1859)。 受还原论思维的影响, 人们总是将高层级的、复杂 的对象分解为较低层级的、简单的对象来处理。应 用于多物种共存的研究, 人们首先将复杂群落系统 分解还原为简单的两两物种之间的相互作用(配对 相互作用), 然后再将这些配对组装起来重新认识 原先的复杂系统。在这个“一分一合”的过程中, 诸 多与复杂系统相关的信息随之丢失, 其中最为重要 的一个方面即为生物间的间接相互作用。这是因为 直接相互作用无法考虑除成对物种以外的其他有 机体的影响, 而在复杂系统里, 有机体常常存在于 相互作用的网络并彼此交织在一起(图1)。另一方面, 人们因为理论模型在考虑间接相互作用之后变得 过于复杂并认为间接相互作用的强度可能很弱, 而 常常在研究中忽略间接相互作用。

复杂系统中忽略间接相互作用会导致至少两 个方面的重要不足: (1)无法全面深入理解物种共存 和生物多样性维持的机制, 配对相互作用的实验和 理论工作很难外推至群落水平; (2)无法准确预测多 样性如何响应生物/非生物环境变化, 其中间接相 互作用是此类预测的关键不确定来源。理论工作和 微宇宙实验证实了间接相互作用的普遍性和对群 落及生态系统功能的重要性(Kerr et al, 2002; van Veen et al, 2005; Soliveres et al, 2015; Bairey et al, 2016; Gallien et al, 2017; Grilli et al, 2017; Letten \& Stouffer, 2019), 然而鲜有来自复杂真实系统的研 究。正如Levine等(2017)所言, 生态学极少有哪个方
面能像间接相互作用这般将革新人们对生物多样 性维持和分布机制的认识。明确考虑间接相互作用 将有效完善和丰富群落生态学的理论框架, 夯实生 态系统生态学的群落学基础。

基于Lotka-Volterra模型, 本文首先介绍了两类 不同的间接相互作用: 链式相互作用和高阶相互作 用。由于链式相互作用本质上还是配对相互作用, 因此我们重点介绍高阶相互作用, 包括跨营养级和 同一营养级内高阶相互作用的研究历史和概况以 及高阶相互作用定义的演变(种群水平), 进而介绍 最新发展的基于个体的高阶相互作用(个体水平), 最后提出间接相互作用未来可能的研究重点和难 点, 尤其是高阶相互作用的内在机理以及与功能性 状之间的可能联系。

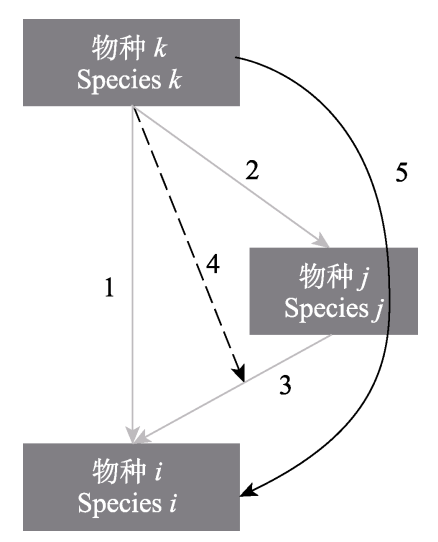

图1 包含直接和间接相互作用的生态网络。灰色箭头为直 接配对相互作用(箭头1-3), 黑色箭头为间接相互作用(箭头 4-5)。在间接相互作用中, 箭头 4 表示高阶相互作用, 即物种 $k$ 影响的是物种 $j$ 和 $i$ 之间的相互作用, 箭头 5 表示链式相互作 用, 即物种 $k$ 先影响物种 $j$ 的密度进而影响物种 $i$ 。可见, 物种 $k$ 对物种 $i$ 既存在直接相互作用 (箭头 1 ), 也存在间接相互作用 (箭头 4 和5)。箭头表示作用方向, 为简单起见, 只绘出了单 向作用。

Fig. 1 The ecological network including direct (arrows 1-3) and indirect interactions (arrows 4-5) between species. Arrow 4 indicates that species $k$ may indirectly affect species $i$ by modifying the per capita effect of species $j$ on species $i$ (higher-order interactions, HOIs). Arrow 5 indicates that species $k$ may indirectly affect species $i$ by changing population density of species $j$ (interaction chains). Therefore, species $k$ may have both direct (arrow 1) and indirect (arrows 4-5) effects on species $i$. For simplicity, we only display direct and indirect effects of species $j$ and $k$ on species $i$. 


\section{间接相互作用}

相比两两物种的直接相互作用, 间接相互作用 指一个物种通过中间物种对目标物种产生的间接 影响, 因此不包括通过非生物因子对目标物种产生 的间接影响(Wootton, 1994a; Abrams, 1995)。间接相 互作用按照中间物种的介导方式可分为两类：(1)密 度介导的间接相互作用(density-mediated indirect interactions, Abrams, 1995), 如物种 $k$ 通过改变物种 $j$ 的密度对物种 $i$ 产生的间接相互作用(图1箭头5), 又 称为链式相互作用(interaction chains); (2)性状介导 的间接相互作用(trait-mediated indirect interactions, Abrams, 1995; Werner \& Peacor, 2003), 如物种 $k$ 通 过改变物种 $j$ 的性状而改变物种 $j$ 对物种 $i$ 的直接作用 强度(图1箭头4), 也称高阶相互作用 (higher-order interactions, HOIs)。Lotka-Volterra模型 (简称L-V模 型)作为生态学里最为重要的经典模型, 可以描述 绝大多数物种间的相互作用, 间接相互作用也不例 外。在经典的多物种 $\mathrm{L}-\mathrm{V}$ 模型中, 物种 $i$ 的单位种群 增长率是所有物种 (包括 $i$ 本身) 密度的线性函数 (Chesson, 2012):

$$
\frac{1}{N_{i}} \frac{d N_{i}}{d t}=r_{i}\left(1-\sum_{j=1}^{S} \alpha_{i j} N_{j}\right)
$$

其中 $r_{i}$ 是物种 $i$ 的内禀增长率, $S$ 是群落中的物种数, $\alpha_{i j}$ 是物种 $j$ 对物种 $i$ 的直接作用强度, $N_{j}$ 是物种 $j$ 的种 群密度。故 $\alpha_{i j} N_{j}$ 项是物种 $j$ 对物种 $i$ 直接作用的总和, 其中物种 $k$ 通过物种 $j$ 对物种 $i$ 产生间接相互作用, 就 是通过改变其中的 $N_{j}$ (链式相互作用, 图1箭头5) 或者 $\alpha_{i j}$ (高阶相互作用, 图1箭头4)引起的。

\section{1 链式相互作用}

在经典的多物种 $\mathrm{L}-\mathrm{V}$ 模型中, 链式相互作用是 普遍存在的, 因为链式相互作用本质上仍然是配对 物种相互作用的迭代和延伸(Levine et al, 2017)。以 图1的三物种群落为例，在不考虑高阶相互作用时 该群落的动态方程是:

$$
\left\{\begin{array}{l}
\frac{1}{N_{i}} \frac{d N_{i}}{d t}=r_{i}\left(1-\alpha_{i i} N_{i}-\alpha_{i j} N_{j}-\alpha_{i k} N_{k}\right) \\
\frac{1}{N_{j}} \frac{d N_{j}}{d t}=r_{j}\left(1-\alpha_{j i} N_{i}-\alpha_{j j} N_{j}-\alpha_{j k} N_{k}\right) \\
\frac{1}{N_{k}} \frac{d N_{k}}{d t}=r_{k}\left(1-\alpha_{k i} N_{i}-\alpha_{k j} N_{j}-\alpha_{k k} N_{k}\right)
\end{array}\right.
$$

在等式(2.1)中，物种 $i$ 在 $t$ 时刻的单位种群增长 速率受其自身及其竞争者(物种 $j$ 和物种 $k$ ) 的影响, 其中物种 $j$ 的密度 $N_{j}$ 由 $t$ 时间内的 $d N_{j}$ 累积而成, 而 $d N_{j}$ 又受物种 $k$ 的密度制约 (当然也受物种 $i$ 和 $j$ 的密度制 约，等式2.2)。因此在同时考虑多物种种群动态的 $\mathrm{L}-\mathrm{V}$ 模型中隐含着这样一条相互作用链: 物种 $k$ 通过 改变物种 $j$ 的密度间接影响物种 $i$ 的种群增长 $\left(N_{k} \rightarrow N_{j}\right.$ $\rightarrow N_{i}$, 图1箭头5)。同理可知 L- $\mathrm{V}$ 模型(等式2)中还包 含有其他的相互作用链(如 $N_{k} \rightarrow N_{i} \rightarrow N_{j}, N_{j} \rightarrow N_{k} \rightarrow N_{i}$ 等)。链式相互作用的形式十分多样，由L-V模型中 的相互作用矩阵A (种内和种间相互作用系数组成 的矩阵，等式3)可以体现，不同形式的链式相互作 用对群落动态的影响也不同(Stouffer \& Bascompte, 2010; Soliveres et al, 2018; Losapio et al, 2019)。

$$
\mathbf{A}=\left(\begin{array}{ccc}
\alpha_{i i} & \alpha_{i j} & \alpha_{i k} \\
\alpha_{j i} & \alpha_{i j} & \alpha_{j k} \\
\alpha_{k i} & \alpha_{k j} & \alpha_{k k}
\end{array}\right)
$$

相互作用矩阵A变化形式复杂多样, 我们这里 仅以两种特殊情形 $\left(\mathrm{A}_{1}\right.$ 和 $\left.\mathrm{A}_{2}\right)$ 为例展示其对群落动态 影响的差异。

$$
\mathbf{A}_{1}=\left(\begin{array}{ccc}
0.2 & 0 & 0 \\
0.3 & 0.2 & 0 \\
0.3 & 0.3 & 0.2
\end{array}\right) \quad \mathbf{A}_{2}=\left(\begin{array}{ccc}
0.2 & 0 & 0.3 \\
0.3 & 0.2 & 0 \\
0 & 0.3 & 0.2
\end{array}\right)
$$

在 $\mathrm{A}_{1}$ 情况下, 物种的竞争优势为: $i>j>k$ 且 $i>$ $k$, 即物种 $i$ 是最强的竞争者, $j$ 次之, $k$ 最弱, 最终物 种 $i$ 会竞争排除掉物种 $j$ 和 $k$, 这种链式相互作用被称 为传递性竞争(transitive competition, Gallien et al, 2017)。在 $\mathrm{A}_{2}$ 情况下, 物种的竞争优势为: $i>j>k>i$, 即三者中没有最强的竞争者，而是呈现一种类似于 剪刀-石头-布的相互制约(当 $i$ 的种群密度增加时, 会抑制 $j$ 的种群增长，进而缓解了 $j$ 对 $k$ 的竞争，最后 $k$ 对 $i$ 的抑制增强, 使 $i$ 的变化趋向于稳定), 使得物种 $i 、 j$ 和 $k$ 虽不能两两共存，但三者可以同时共存，这 种链式相互作用被称为非传递性竞争(intransitive competition, Gallien et al, 2017)。非传递性竞争所带 来的这种制约回环，已被证明可以促进多物种共存 (Kerr et al, 2002; Reichenbach et al, 2007; Allesina \& Levine, 2011; Rojas-Echenique \& Allesina, 2011), 而 且群落的稳定性与非传递性环的出现频率、数量和 长度呈正相关(Laird \& Schamp, 2006, 2008; Gallien 
et al, 2017)。由于非传递性环广泛存在于多种生物 类群中(Soliveres et al, 2018), 这种链式相互作用模 式还可能显著影响物种多样性和生态系统功能 (Soliveres et al, 2015; Maynard et al, 2017)。除了在同 一营养级的链式相互作用, 食物网中的链式相互作 用也是长期被研究者关注的。例如食物网中特定的 相互作用模式(motif), 包括食物链(food chain)、似 然竞争(apparent competition)及杂食环(omnivory), 它们在自然界的出现频率和对食物网稳定性的影 响都有显著区别 (Bascompte \& Melián，2005; Stouffer \& Bascompte, 2010; 徐光华等, 2019; 王少 鹏，2020)。除了特定的相互作用模式之外, 基于直 接相互作用网络所构成的整体拓扑结构, 也可以揭 示链式相互作用对群落的可能影响(方强和黄双全, 2012; 宋础良, 2020)。例如Bastolla等(2009)证明互 惠网络的嵌套性(nestedness) 可以使物种具有更多 重合的互惠合作者，进而最大化由合作者传递的间 接促进作用, 从而可以减小物种之间的竞争强度并 且维持物种多样性。

\section{2 高阶相互作用}

相比于链式相互作用与直接配对相互作用的 密切联系(同属于物种密度 $N$ 的改变), 高阶相互作 用的特点就在于两两物种间的直接相互作用强度 不是恒定的, 而是受其他物种调节(图1箭头4)。在图 1 中，假定物种 $k$ 平均每个个体对 $\alpha_{i j}$ (物种 $j$ 对物种 $i$ 的 直接作用强度)的改变强度为 $\beta_{i j, k}$, 则物种 $j$ 对物种 $i$ 的直接作用强度因物种 $k$ 存在的改变强度 $\left(\Delta \alpha_{i j}\right)$ 为:

$$
\Delta\left(\alpha_{i j}\right)=\beta_{i j, k} N_{k}
$$

从而物种 $k$ 通过调节物种 $j$ 的性状对物种 $i$ 的单位种 群增长速率的改变为:

$$
\Delta\left(\frac{1}{N_{i}} \frac{d N_{i}}{d t}\right)=-r_{i} \beta_{i j, k} N_{j} N_{k}
$$

链式相互作用(物种 $k$ 通过改变物种 $j$ 的密度对 物种 $i$ 的间接作用)的发生具有时滞性(time lag), 而 高阶相互作用 (物种 $k$ 通过改变物种 $j$ 的性状对物种 $i$ 的间接作用)的发生是即时的(immediateness)。在高 阶相互作用存在的情况下, 群落的动态变得更加复 杂和不可预测(Wootton, 1994a), 包含高阶相互作用 模型的复杂度与物种数的幂指数(平方、立方等)成 正比。事实上, 自然群落的动态复杂性确实超出直 接相互作用与链式相互作用所预测的范围
(Mayfield \& Stouffer, 2017)。由于高阶相互作用的现 象在群落中十分普遍(Wootton, 1994a), 并且其相互 作用强度已被证明并不小于直接相互作用(Werner \& Peacor, 2003), 所以接下来着重介绍高阶相互作 用这一非常重要却又长期被忽略的相互作用类型。

\section{高阶相互作用研究的发展脉络}

由于高阶相互作用的定义和相关术语的使用 较为混淆, 这里我们简要整理了它们之间的关系 (图2)。传统意义上的高阶相互作用, 一般又称为相 互作用的调节(interaction modifications, Case \& Bender, 1981; Abrams, 1983; Adler \& Morris, 1994; Levine et al, 2017), 是指一个物种对另一个物种的 直接作用强度受到其他物种的影响。其发生机制在 于物种 $j$ 在物种 $k$ 存在时有性状(形态、生理、行为等) 上的可塑性变化, 并且物种 $j$ 的这种可塑性变化会 改变其对物种 $i$ 的直接作用强度(图1箭头 4 ), 因而又 称为性状介导的间接相互作用(Abrams，1995; Werner \& Peacor, 2003; Levine et al, 2017)。因此这 类定义一般认为高阶相互作用只可能发生在由三 个或三个以上物种所组成的系统中，不过也有研究 认为可发生在两物种的情况(物种 $j$ 或者物种 $k$ 可与 目标物种 $i$ 为同一物种) (Case \& Bender, 1981; Kleinhesselink et al, 2019)。近年来相关研究将高阶 相互作用定义为系统中所有物种(包括目标物种自 身)对目标物种单位种群增长速率的非线性密度制 约效应(nonlinear density dependence, Bairey et al, 2016; Kleinhesselink et al, 2019; Letten \& Stouffer, 2019; Xiao et al, 2020)。Kleinhesselink等(2019)将这 两类定义区分为狭义高阶相互作用(hard-HOIs，前 者)和广义高阶相互作用(soft-HOIs，后者)。狭义的 高阶相互作用因一个物种对另一个物种的直接作 用强度依赖于其他物种，一定会产生非线性密度制 约效应，因而属于广义高阶相互作用的范畴。而广 义高阶相互作用不仅包含狭义相互作用，还包含种 内高阶相互作用 (intraspecific HOIs)或种内非线性 (intraspecific nonlinearity), 即 $\beta_{i j, j} N_{j}^{2}$ 项(等式5物种 $j$ 和 $k$ 为同一物种时)。传统的研究强调狭义与广义相 互作用的区分，并提出了一系列检验种群动态模型 中是否包含狭义高阶相互作用的方法(表1)。我们则 认为狭义与广义的高阶相互作用可统一于个体水 平的高阶相互作用(individual-level HOIs), 详见下 


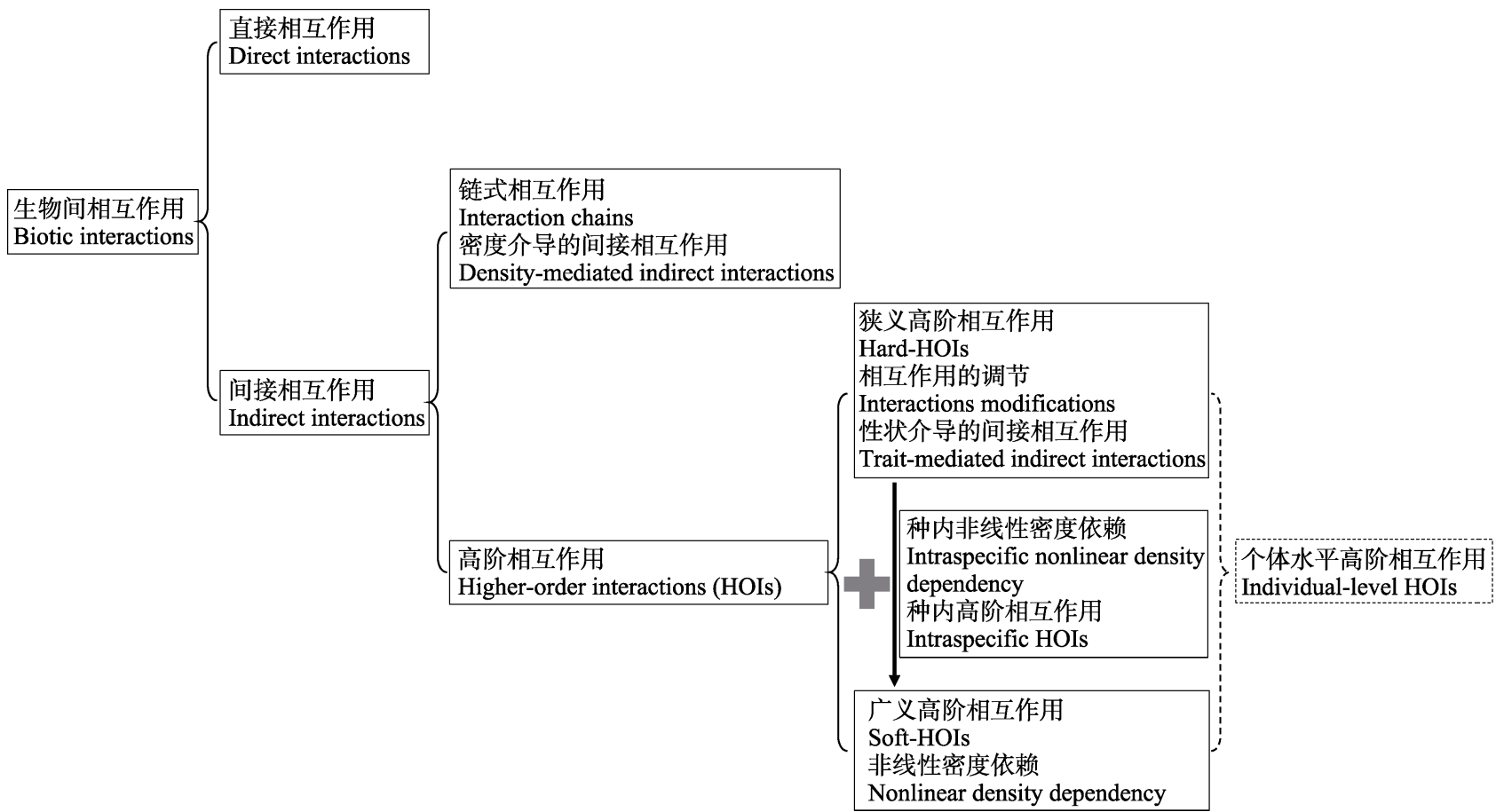

图2 生物间相互作用的类型和关系。同一方框内的不同术语为不同角度描述的同一类型的相互作用，虚线部分为作者见解， 尚无相关文献明确说明。

Fig. 2 The types of biotic interactions. The different terms in the same box were used to describe the same type of interaction in different studies. The part in dashed line is our own opinion.

文第 3 节。

\section{1 跨营养级的高阶相互作用}

在食物链/网研究中，高阶相互作用通常被称 为性状介导的间接相互作用。Werner和Peacor (2003) 以及朱玉等(2017)详细回顾了性状介导的间接相互 作用的特征、发生机制、作用途径及实验证据, 并 根据性状将其分为通过行为、生理、形态、发育以 及生活史等介导的间接相互作用。现有研究主要关 注的是行为和生理性状, 而关于形态、发育以及生 活史等方面的性状的研究实例并不多(e.g. Mopper et al, 1991; Tscharntke, 1999; van Veen \& Godfray, 2013; Xi et al, 2016)。在行为性状方面, 处于食物链 中间的物种在捕食风险和能量摄入两方面存在权 衡, 因此其采食行为同时受到上、下营养级物种关 系的调控。面对捕食者时要增加躲避时间进而能量 摄入减少，而当处于较低的资源水平时采食活动更 加频繁，被捕食风险增大(Huang \& Sih，1990; Johansson, 1995; Beckerman et al, 1997; Lima, 1998)。另一类基于行为介导的间接相互作用是通过 生境利用的转变 (Messina, 1981; Werner et al, 1983; Werner \& Gilliam, 1984; Wootton, 1993; Turner, 1996,
1997)。如当捕食者大口黑鲈(Micropterus salmoides) 存在时, 蓝鳃太阳鱼(Lepomis macrochirus)对生境 的利用从池塘中心逐渐转移至池塘沿岸植被区域, 导致池塘中心浮游动物丰度显著增加(Turner \& Mittelbach, 1990)。关于生理性状的研究主要集中在 植物的诱导性防御反应。例如，美国赤杨(Alnus rubra) 在被采食后产生防御反应, 通过降低叶片氮 含量进而提高碳氮比，减少了陆生植食者和水生分 解者对其叶片的消耗(Jackrel \& Wootton, 2015)。近 年在四川红原高寒草甸的野外观测发现，菊科植物 的花序对拟寄生蜂寄生实蝇有明显的限制和选择 作用, 即高阶专化作用(higher-order specialization, Xi et al, 2017)。Liao等(2020)通过对菊科植物一石蝇 -拟寄生蜂三分网络的模拟分析发现，高阶专化作 用提升了石蝇物种多样性, 但降低了菊科植物和寄 生蜂的物种丰富度, 强调了来自多分网络的不同物 种之间的高阶相互作用对物种多样性维持的重要 性。此外, 一些依赖动物扩散种子的植物会通过大 年结实调控动物咜食行为而提高种子扩散效率, 因此动物行为的变化可以介导植物间的相互作用 关系, 进而影响植物共存(Yang et al, 2020; 杨锡福 
表1 检验模型是否包含狭义高阶相互作用的方法及部分常见模型检验结果。、表示模型中包含狭义高阶相互作用(模型不满 足方法中等式), $x$ 表示模型不含狭义高阶相互作用(模型满足方法中等式)。这些方法旨在将狭义高阶相互作用从广义高阶相互 作用中区分出来, 其中(1)、(3)和(5)用于检验两物种或两物种以上系统是否包含狭义高阶相互作用，(2)和(4)分别是(1)和(3)用 于将狭义高阶相互作用严格定义在三物种或三物种以上系统中时的情况, 因而方法(1)和(2), (3)和(4)在三个以上物种组成的 系统中等效。 $F_{i}$ 表示物种 $i$ 的单位种群增长率是其自身及竞争者密度的函数, 这里给出几个常见模型的 $F_{i}$ 函数表达式。如果函 数 $F_{i}$ 对 $N_{j}$ 的偏导数 $\partial F_{i} / \partial N_{j}$ 能表达成 $N_{j}$ 的函数 $G_{i j}\left(N_{j}\right)$ (方法1), 或是 $N_{i}$ 和 $N_{j}$ 的函数 $G_{i j}\left(N_{i}, N_{j}\right)($ 方法 2$)$, 或是 $N_{j}$ 和 $F_{i}$ 自身的函数 $G_{i j}\left(F_{i}, N_{j}\right)$ (方法3), 或是 $N_{i}$ 和 $N_{j}$ 以及 $F_{i}$ 的函数 $G_{i j}\left(F_{i}, N_{i}, N_{j}\right)$ (方法4), 则模型没有狭义高阶相互作用。方法 $(5)$ 中, $\Theta_{i}$ 表示函数 $F_{i}$ 中所有参数的集合, $\varphi_{i j}$ 表示除物种 $j$ 外所有物种的密度均为 0 时函数 $F_{i}\left(0, \ldots, N_{j}, \ldots, 0\right)$ 中的参数, $\Phi_{i}$ 则是 $\varphi_{i j}(j=1, \ldots, S)$ 的并集。

若 $\Theta_{i}=\Phi_{i}$, 则模型没有狭义高阶相互作用。

Table 1 The methods of detecting whether a species interaction model contains hard higher-order interactions (hard-HOIs) and the outcomes of some well known models. $\checkmark$ and $\times$ indicate the model contains (the equation in a method is violated) and does not contain (the equation in a mothed is satisfied) hard-HOIs, respectively. Methods (1), (3) and (5) are used in the case of HOIs defined in systems of two or more species, and methods (2) and (4) are special cases of (1) and (3) where HOIs are strictedly defined in systems of three or more species. $F_{i}$ indicates the per capita growth rate of species $i$ as a function the densities of itself and its competitiors. If the partial derivative of $F_{i}$ to $N_{j}\left(\partial F_{i} / \partial N_{j}\right)$ can be expressed as only a function of $N_{j}: G_{i j}\left(N_{j}\right)$ (method 1$)$, or a function of $N_{i}$ and $N_{j}: G_{i j}\left(N_{i}, N_{j}\right)$ (method 2), or function of $N_{j}$ and $F_{i}: G_{i j}\left(F_{i}, N_{j}\right)$ (method 3), or a function of $N_{i}, N_{j}$ and $F_{i}: G_{i j}\left(F_{i}, N_{i}, N_{j}\right)$ (method 4), then the model $F_{i}$ does not contain hard-HOIs according to methods $1-4$, respectively. In method (5), $\Theta_{i}$ indicates the set of paramters in function $F_{i}$; $\varphi_{i j}$ indicates the set of parameters in function $F_{i}\left(0, \ldots, N_{j}, \ldots, 0\right)$ when densities of all species are zero except species $j$; $\Phi_{i}$ is the union of $\varphi_{i j}\left(j=1, \ldots\right.$, S). If $\Theta_{i}=\Phi_{i}$, then the model $F_{i}$ does not contain hard-HOIs.

\begin{tabular}{|c|c|c|c|c|c|}
\hline $\begin{array}{l}\text { 模型 } \\
\text { Models }\end{array}$ & & & $\begin{array}{l}\text { 检验方; } \\
\text { Method }\end{array}$ & & \\
\hline $\begin{array}{l}\frac{1}{N_{i}} \frac{d N_{i}}{d t}=F_{i}\left(N_{1}, \ldots, N_{j}, \ldots, N_{s}\right) \\
\frac{N_{i}(t+1)}{N_{i}(t)}=F_{i}\left(N_{1}, \ldots, N_{j}, \ldots, N_{s}\right)\end{array}$ & $\begin{array}{l}\text { (1) } \frac{\partial F_{i}}{\partial N_{j}}=G_{i j}\left(N_{j}\right) \\
\text { (Case \& Bender, 1981) }\end{array}$ & $\begin{array}{l}\text { (2) } \frac{\partial F_{i}}{\partial N_{j}}=G_{i j}\left(N_{i}, N_{j}\right) \\
\text { (Billick \& Case, 1994) }\end{array}$ & $\begin{array}{l}\text { (3) } \frac{\partial F_{i}}{\partial N_{j}}=G_{i j}\left(F_{i}, N_{j}\right) \\
\text { (Adler \& Morris, 1994) }\end{array}$ & $\begin{array}{l}\text { (4) } \frac{\partial F_{i}}{\partial N_{j}}=G_{i j}\left(F_{i}, N_{i}, N_{j}\right) \\
\text { (Adler \& Morris 1994) }\end{array}$ & 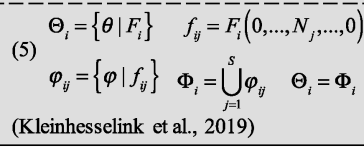 \\
\hline $\begin{array}{l}F_{i}=r_{i}\left(1-\sum_{j=1}^{S} \alpha_{i j} N_{j}\right) \\
\text { L-V Model (Chesson, 2012) }\end{array}$ & $x$ & $x$ & $x$ & $x$ & $x$ \\
\hline $\begin{array}{l}F_{i}=r_{i}\left(1+\sum_{j=1}^{S} \alpha_{i j} N_{j}\right)^{-\theta_{i}} \\
\text { (Hassel \& Comins, 1976) }\end{array}$ & $\sqrt{ }$ & $\sqrt{ }$ & $x$ & $x$ & $x$ \\
\hline $\begin{array}{l}F_{i}=r_{i} \exp \left(-\sum_{j=1}^{S} \alpha_{i j} N_{j}\right) \\
\text { (Ricker, 1958) }\end{array}$ & $\sqrt{ }$ & $\sqrt{ }$ & $x$ & $x$ & $x$ \\
\hline $\begin{array}{l}F_{i}=r_{i}\left(1-\sum_{j=1}^{s} \alpha_{i j} N_{j}-\sum_{j, k} \beta_{i, k} N_{j} N_{k}\right) \\
\text { (Letten \& Stouffer, 2019) }\end{array}$ & $\sqrt{ }$ & $\sqrt{ }$ & $\sqrt{ }$ & $\sqrt{ }$ & $\begin{array}{l}\times(j=k) \\
\sqrt{ }(j \neq k)\end{array}$ \\
\hline$F_{i}=r_{i}\left(\exp \left(-\sum_{j=1}^{S} \alpha_{i j} N_{j}\right)-\sum_{j=1}^{S} \alpha_{i j} N_{j}\right.$ & $\sqrt{ }$ & $\sqrt{ }$ & $\sqrt{ }$ & $\sqrt{ }$ & $x$ \\
\hline
\end{tabular}

等, 2020)。众多的实验证据表明，性状介导的间接 相互作用在水生和陆生系统中均普遍存在, 且对 群落动态有显著的影响。

\section{2 同一营养级的高阶相互作用}

与跨营养级研究类似, 性状介导的间接相互作 用也会导致同一营养级物种之间产生高阶相互作 用。例如, 长叶车前(Plantago lanceolate)会抑制紫 羊茅(Festuca rubra)根系的生长, 进而减弱紫羊茅 对群落内其他物种的竞争强度(Padilla et al, 2013)。 需要特别注意的是, 表象竞争模型 (phenomenological model of competition), 如 L-V 模 型, 通常用竞争系数来描述一个物种对另一个物种 的竞争强度, 而不考虑具体的生态学过程或潜在机 制。基于消费者一资源竞争 (consumer-resource competition) 的机理模型 (mechanistic model of competition)表明, 当资源非 logistic 增长或消费者 对资源密度呈现非线性功能响应时，就会产生高阶 相互作用(Abrams, 1983; Kleinhesselink et al, 2019; Letten \& Stouffer, 2019)。这表明, 高阶相互作用事 实上是表象模型的涌现特征(emergent properties)。 
然而, 无论是考虑表象还是机理竞争模型, 同一营 养级两个物种之间的相互作用在多大程度上受群 落内其他物种的影响仍是一个至关重要的问题。

虽然跨营养级物种之间的高阶相互作用已有 广泛研究, 却鲜有研究在自然群落和实验系统中去 验证同一营养级高阶相互作用的普遍性和相对重 要性。同一营养级高阶相互作用的实验研究可以追 溯到20世纪60年代(Hairston et al, 1968; Vandermeer, 1969), 这一期间的研究主要关注的是高阶相互作 用的检验方法。最初, 人们意识到简单线性的 $\mathrm{L}-\mathrm{V}$ 竞争模型常常无法准确描述和预测物种相互作用 对群落动态的影响, 因而通过在模型中加入高阶交 互项对L-V模型进行扩展(Vandermeer, 1969; Wilbur, 1972; Neill, 1974)。这也是为什么关于同一营养级高 阶相互作用的研究主要集中在种群水平。经典的实 验设计是分别在两两配对以及多物种组合下评估 物种的表现, 检验目标物种对其他物种单独的响应 (配对相互作用)能否预测出目标物种对多物种组合 的响应 (Vandermeer, 1969; Morin et al, 1988; Worthen \& Moore, 1991)。常用的统计检验方法是方 差分析(ANOVA)。但需要注意的是, 对于不同的竞 争模型(包括不同响应变量、数据转换、模型函数形 式等, 描述种群动态和竞争的模型有很多, L-V 模型 只是其中一类), 对应的统计检验方法也可能不同 (Case \& Bender, 1981; Billick \& Case, 1994; Wootton, 1994b)。检验高阶相互作用在自然系统中重要性的 另外一种方法是在配对竞争模型的基础上纳入高 阶相互作用的影响, 对实验或自然群落观测数据进 行统计拟合(Weigelt et al, 2007; Mayfield \& Stouffer, 2017)。量化高阶相互作用是理解高阶相互作用重要 性的第一步，目前依然是一个巨大的挑战。

\section{3 基于个体的高阶相互作用}

近年来高阶相互作用的研究主要是在经典的 $\mathrm{L}-\mathrm{V}$ 模型中(仅考虑直接相互作用)引入密度制约的 高阶项, 然后通过模拟比较种群动态在考虑高阶相 互作用后与经典模型的差异, 进而探讨其对物种共 存和生物多样性的影响(Bairey et al, 2016; Letten \& Stouffer, 2019; Singh \& Baruah, 2020)。一方面, 种 群的动态变化归根结底是个体的存活、生长和繁殖 的过程 (不考虑迁入、迁出), 因而未来高阶相互作 用的研究需要更加关注其对个体适合度(存活、生长
和繁殖) 的影响。Mayfield和Stouffer (2017)首次在 一年生草本植物群落中探索了高阶相互作用对个 体种子数量的影响。另一方面, 广义高阶相互作用 中的种内非线性虽不属于传统的狭义高阶相互作 用的范畴，但其本质上可以解释为个体水平的相互 作用的调节：物种 $i$ 的一个个体对另一个个体的直 接作用强度是依赖于该物种其他个体的。因此，广 义高阶相互作用和狭义高阶相互作用实际上可统 一于个体水平的高阶相互作用(图2虚线部分)。尤为 重要的是, 个体水平的高阶相互作用可以明确将个 体间的差异(如个体大小、个体的空间分布)考虑进 来, 对研究诸如森林群落中的直接与高阶相互作用 具有非常重要的意义(Hegyi，1974; Canham et al, 2004; Uriarte et al, 2004; Hasenauer, 2006)。以森林群 落为例, $\mathrm{Li}$ 等 $(2020)$ 最近提出了在量化邻体 $(N$ 个邻 体分属 $S$ 个物种, $N=\sum_{j=1}^{s} N_{j}, N_{j}$ 是物种 $j$ 的多度)对目 标个体 $i_{m}$ 的直接与高阶相互作用中考虑个体大小与 空间分布的一种新方法(图3)。 $N$ 个邻体对目标个体 $i_{m}$ 的直接相互作用 $\left(D I_{i_{m}} \mid[N]\right)$ 是每个邻体对目标个体 直接相互作用之和(图3直线箭头), 并假定邻体 $j_{p}$ 对 目标个体 $i_{m}$ 的直接相互作用强度 $\left(\alpha_{i_{m} j_{p}}\right)$ 与邻体大小 (用邻体胸径 $D B H$ 度量)成正比而与邻体到目标个体 的距离 $\left(d\left[i_{m}, j_{p}\right]\right)$ 成反比，且这种直接相互作用仅发 生于邻体在目标个体给定半径为 $R$ 的邻域内:

$$
D I_{i_{m}} \mid[N]=\sum_{j=1}^{S} \sum_{p=1}^{N_{j}} \alpha_{i_{m} j_{p}}=\sum_{j=1}^{S} \alpha_{i j} \cdot\left(\sum_{p=1}^{N_{j}} \frac{D B H_{j_{p}}^{u}}{d\left[i_{m}, j_{p}\right]^{v}}\right)
$$

为计算方便，将 $d\left[i_{m}, j_{p}\right]$ 大于邻域半径 $R$ 的这些 距离设置成无穷大以去掉邻域外个体对目标个体 的影响。 $N$ 邻体对目标个体的高阶相互作用是每个 邻体通过其他邻体对目标个体高阶相互作用之和 (图3曲线箭头), 并且邻体 $k_{q}$ 通过邻体 $j_{p}$ 对目标个体 $i_{m}$ 的高阶相互作用 $\left(\beta_{i_{m} j_{p}, k_{q}}\right)$ 取决于邻体 $k_{q}$ 对邻体 $j_{p}$ 的 直接相互作用强度 $\left(\alpha_{j_{p} k_{q}}\right)$ 以及邻体 $j_{p}$ 对目标个体 $i_{m}$ 的直接相互作用强度 $\left(\alpha_{i_{m_{j}}}\right)$ :

$$
\begin{aligned}
& H O I_{i_{m}} \mid[N]=\sum_{j=1}^{S} \sum_{k=1}^{S} \sum_{p=1}^{N_{j}} \sum_{q=1}^{N_{k}} \beta_{i_{m} j_{p}, k_{q}}= \\
& \sum_{j=1}^{S} \sum_{k=1}^{S} \beta_{i j, k} \cdot\left(\sum_{p=1}^{N_{j}} \sum_{q=1}^{N_{k}} \frac{D B H_{j_{p}}^{u}}{d\left[i_{m}, j_{p}\right]^{v}} \cdot \frac{D B H_{k_{q}}^{u}}{d\left[j_{p}, k_{q}\right]^{v}}\right)
\end{aligned}
$$

同样地, 如果 $d\left[i_{m}, j_{p}\right]$ 或 $d\left[j_{p}, k_{q}\right]$ 大于邻域半径 $R$, 就 


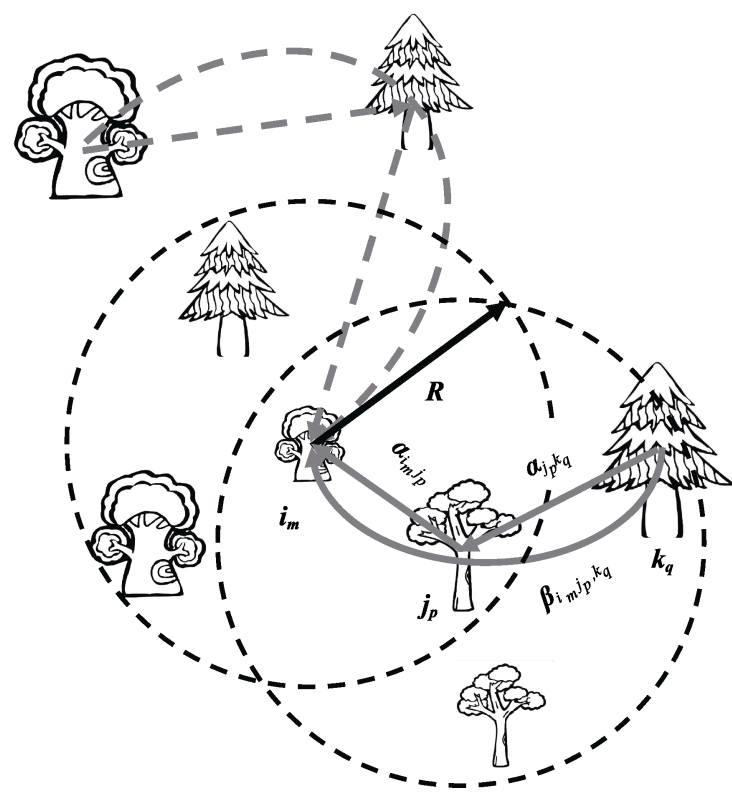

图3 邻体对目标个体的直接(直线箭头)与高阶相互作用(曲 线箭头)。参数 $\alpha_{i m j_{p}}$ 表示的是邻体 $j_{p}$ 对目标个体 $i_{m}$ 的直接相互 作用, 参数 $\beta_{i_{m} j_{p}, k_{q}}$ 表示的是邻体 $k_{q}$ 通过个体 $j_{p}$ 对目标个体 $i_{m}$ 的 高阶相互作用。森林群落研究中一般假定直接相互作用发生 于邻体 $j_{p}$ 在目标个体 $i_{m}$ 半径为 $R$ 的邻域内 (实直线箭头), 因而 高阶相互作用发生于当邻体 $j_{p}$ 在目标个体 $i_{m}$ 半径为 $R$ 的邻域 内且邻体的邻体 $\boldsymbol{k}_{q}$ 在邻体 $j_{p}$ 的邻域内(实曲线箭头)。虚线箭 头表示邻域外不需要考虑的直接与高阶相互作用。

Fig. 3 Direct (straight arrows) and higher-order interactions (curve arrows) of neighbouring trees on a focal tree. The parameter $\alpha_{i_{m j p}}$ quantifies the direct effect of a neighbour (individual $p$ of species $j$ ) on the focal tree (individual $m$ of species $i$ ). The direct interaction occurs only when a neighbour $\left(j_{p}\right)$ is located within a maximum radius $(R)$ of $i_{m}$ (solid straight arrows). The parameter $\beta_{i m j p, k_{q}}$ quantifies the higher-order effect of a neighbour (individual $q$ of species $k$ ) on the focal tree through another neighbour (individual $p$ of species $j$ ). Higher-order interaction occurs only $j_{p}$ is located within the maximum radius $(R)$ of $i_{m}$ and $k_{q}$ is located within the maximum radius $(R)$ of $j_{p}$ (solid curve arrows). Dashed arrows indicate direct interactions and higher-order interactions that are not considered when a neighbour is located outside the maximum radius $(R)$ of the focal tree or its neighbour(s).

将其设置成无穷大。在不考虑个体大小与空间距离 时 $(u=0$ 且 $v=0)$, 邻体对目标个体的直接与高阶相 互作用的量化可简化成仅与邻体密度相关的形式 (Mayfield \& Stouffer, 2017)。考虑个体大小与空间分 布时 $(u \neq 0$ 且 $v \neq 0)$ 的量化方法可区分邻体 $k_{q}$ 通过 邻体 $j_{p}$ 对目标个体 $i_{m}$ 的高阶相互作用 $\left(\beta_{i_{m} j_{p}, k_{g}}\right)$ 和邻体 $j_{p}$ 通过邻体 $k_{q}$ 对目标个体 $i_{m}$ 的高阶相互作进而区分 物种 $k$ 通过物种 $j$ 对物种 $i$ 的高阶相互作用 $\left(\beta_{i j, k}\right)$ 和物 种 $j$ 通过物种 $k$ 对物种 $i$ 的高阶相互作用 $\left(\beta_{i k, j}\right)$, 这是仅
在种群水平上量化高阶相互作用所无法实现的。

\section{研究展望}

4.1 检验自然群落中高阶相互作用的普遍性与相 对重要性

尽管最近的研究从理论层面上证明了高阶相 互作用对物种共存与物种多样性维持的重要意义 (Bairey et al, 2016; Grilli et al, 2017; Letten \& Stouffer, 2019; Singh \& Baruah, 2020), 然而鲜有研 究在自然植物群落中去验证同一营养级内高阶相 互作用的普遍性和相对重要性。Mayfield 和 Stouffer (2017)研究发现在一年生草本植物群落中，包含高 阶相互作用的模型显著提高了对个体产生种子数 量的解释度，从而首次证实高阶相互作用在自然群 落中的重要性。今后的研究需要在更多不同的自然 群落中采用类似于第 3 节中介绍的量化个体水平高 阶相互作用的方法，去检验高阶相互作用对个体适 合度(存活、生长和繁殖)的影响。同时，由于这种量 化高阶相互作用的方法中的参数数量与物种数的 平方成正比，因此很难直接适用于物种丰富度较高 的群落。最近已有研究表明将邻体按生活型或个体 大小等分成数量较少的类群后(而非按物种分类), 再采用这种方法量化高阶相互作用，既可简化模型 的复杂度又能提高模型解释度和预测能力( $\mathrm{Li}$ et al, 2020; Martyn et al, 2020)。在森林群落中，已有许多 工作研究邻体直接相互作用对目标个体的存活和 生长的影响(Hegyi，1974; Lorimer, 1983; Wykoff, 1990; Monserud \& Sterba, 1999; Canham et al, 2004; Uriarte et al, 2004; Hasenauer, 2006), 然而到目前为 止却未见研究邻体高阶相互作用在其中所起作用 的文献。基于固定监测样地的多次普查数据使得检 验不同森林群落中邻体高阶相互作用对目标个体 存活和生长的相对重要性成为可能。在此基础上, 可进一步在全球尺度上研究森林群落中高阶相互 作用的纬度梯度格局, 并推断潜在的生物(多样性 等)和非生物影响因素(气候、地形和土壤等)。

\section{2 揭示高阶相互作用的内在机制}

通过上述自然群落的观察研究(用包含高阶相 互作用的模型拟合自然群落的观测数据)可探索高 阶相互作用的普遍性和相对重要性(Where, When and What), 但难以揭示高阶相互作用发生的内在机 制(How and Why) (Letten \& Stouffer, 2019)。在植物 
群落中鲜有单种(无邻体, 无相互作用)与双种(一个 邻体, 仅存在直接相互作用)的情况, 故而需要通过 控制实验的手段, 比较它们和多种(多个邻体, 直接 和高阶相互作用)情况下个体存活、生长和繁殖的差 异, 为个体水平高阶相互作用提供更直接有力的证 据。并在此基础上建立高阶相互作用强度与目标个 体在有无邻体情况下性状的可塑性变化强度的联 系，从而揭示高阶相互作用性状介导的发生机制 (高阶相互作用主要是通过哪种性状的可塑性变化 所介导的, 这种可塑性变化的强度和方向如何, 高 阶相互作用是放大还是抑制直接相互作用)。如邻体 $k_{q}$ 通过邻体 $j_{p}$ 对目标个体 $i_{m}$ 的高阶相互作用, 可能是 由于邻体 $k_{q}$ 通过抑制邻体 $j_{p}$ 的根系生长, 从而抑制 邻体 $j_{p}$ 对目标个体 $i_{m}$ 的直接作用强度(抑制 $j_{p}$ 对目标 个体 $i_{m}$ 根系生长的抑制)。在上述实验的基础上，进 行杀菌和去除植食性昆虫等处理, 可进一步研究多 营养级之间高阶相互作用发生的内在机制。此外, 探索竞争相互作用的机理模型, 通过明确包含资源 或者捕食者的动态, 有助于预测什么情况下高阶相 互作用可能出现及对种群动态、群落结构和生态系 统功能的影响 (Abrams，1983; Letten \& Stouffer, 2019)。

\section{3 高阶相互作用对多物种共存和生态系统功能 的影响}

揭示高阶相互作用对物种共存的影响，需要进 一步与当代物种共存理论相结合, 探索什么情况下 高阶相互作用会有利于或不利于物种共存，以及所 带来的生态系统水平上的影响。例如, 不同方向和 强度的高阶相互作用如何通过调节种内和种间相 互作用影响物种共存以及竞争网络对物种丧失的 稳健性(Singh \& Baruah, 2020)。此外, 已有的关于生 物多样性和生态系统功能的研究主要集中在同一 个营养级内部, 如植物多样性与生态系统功能关系 的研究 (Tilman et al, 1997; Loreau, 1998, 2000; Hector et al, 1999; Spehn et al, 2005)。近年来，基于 食物网的相关研究发现, 不同营养级的生物多样性 也会对生态系统功能产生复杂的影响(Thébault \& Loreau, 2003; Ives et al, 2005)。在此基础上，一些研 究通过在食物网中引入高阶相互作用, 发现其能影 响生态系统功能以及生物多样性与生态系统功能 之间的关系(Arditi et al, 2005; Goudard \& Loreau, 2008; Lin \& Sutherland, 2014)。然而, 目前还没有研
究关注同一营养级内部高阶相互作用对生态系统 功能的影响。简言之, 将间接相互作用尤其是高阶 相互作用嵌入现有的各类生态学理论框架中, 或许 可以更好地描述和预测人类世背景下的种群动态、 群落结构和生态系统功能。

\section{参考文献}

Abrams PA (1983) Arguments in favor of higher order interactions. The American Naturalist, 121, 887-891.

Abrams PA (1995) Implications of dynamically variable traits for identifying, classifying, and measuring direct and indirect effects in ecological communities. The American Naturalist, 146, 112-134.

Adler FR, Morris WF (1994) A general test for interaction modification. Ecology, 75, 1552-1559.

Allesina S, Levine JM (2011) A competitive network theory of species diversity. Proceedings of the National Academy of Sciences, USA, 108, 5638-5642.

Arditi R, Michalski J, Hirzel AH (2005) Rheagogies: Modelling non-trophic effects in food webs. Ecological Complexity, 2, 249-258.

Bairey E, Kelsic ED, Kishony R (2016) High-order species interactions shape ecosystem diversity. Nature Communications, 7, 12285.

Bascompte J, Melián CJ (2005) Simple trophic modules for complex food webs. Ecology, 86, 2868-2873.

Bastolla U, Fortuna MA, Pascual-García A, Ferrera A, Luque B, Bascompte J (2009) The architecture of mutualistic networks minimizes competition and increases biodiversity. Nature, 458, 1018-1020.

Beckerman AP, Uriarte M, Schmitz OJ (1997) Experimental evidence for a behavior-mediated trophic cascade in a terrestrial food chain. Proceedings of the National Academy of Sciences, USA, 94, 10735-10738.

Billick I, Case TJ (1994) Higher order interactions in ecological communities: What are they and how can they be detected? Ecology, 75, 1529-1543.

Canham CD, LePage PT, Coates KD (2004) A neighborhood analysis of canopy tree competition: Effects of shading versus crowding. Canadian Journal of Forest Research, 34, 778-787.

Case TJ, Bender EA (1981) Testing for higher order interactions. The American Naturalist, 118, 920-929.

Chesson P (2000) Mechanisms of maintenance of species diversity. Annual Review of Ecology and Systematics, 31, 343-366.

Chesson P (2012) Species competition and predation. In: Encyclopedia of Sustainability Science and Technology (ed. Leemans R), pp. 223-256. Springer, New York.

Chesson P (2018) Updates on mechanisms of maintenance of species diversity. Journal of Ecology, 106, 1773-1794. 
Chu CJ, Wang YS, Liu Y, Jiang L, He FL (2017) Advances in species coexistence theory. Biodiversity Science, 25, 345-354. (in Chinese with English abstract) [储诚进, 王酉 石, 刘宇, 蒋林, 何芳良 (2017) 物种共存理论研究进展. 生物多样性, 25, 345-354.]

Darwin C (1859) On the Origin of Species by Means of Natural Selection. John Murray, London.

Fang Q, Huang SQ (2012) Progress in pollination networks: Network structure and dynamics. Biodiversity Science, 20, 300-307. (in Chinese with English abstract) [方强, 黄双全 (2012) 传粉网络的研究进展: 网络的结构和动态. 生物 多样性, 20, 300-307.]

Gallien L, Zimmermann NE, Levine JM, Adler PB (2017) The effects of intransitive competition on coexistence. Ecology Letters, 20, 791-800.

Goudard A, Loreau M (2008) Nontrophic interactions, biodiversity, and ecosystem functioning: An interaction web model. The American Naturalist, 171, 91-106.

Grilli J, Barabás G, Michalska-Smith MJ, Allesina S (2017) Higher-order interactions stabilize dynamics in competitive network models. Nature, 548, 210-213.

Hairston NG, Allan JD, Colwell RK, Futuyma DJ, Howell J, Lubin MD, Mathias J, Vandermeer JH (1968) The relationship between species diversity and stability: An experimental approach with protozoa and bacteria. Ecology, 49, 1091-1101.

Hasenauer H (2006) Sustainable Forest Management: Growth Models for Europe. Springer, New York.

Hassell MP, Comins HN (1976) Discrete time models for two-species competition. Theoretical Population Biology, 9, 202-221.

Hector A, Schmid B, Beierkuhnlein C, Caldeira MC, Diemer M, Dimitrakopoulos PG, Finn JA, Freitas H, Giller PS, Good J, Harris R, Högberg P, Huss-Danell K, Joshi J, Jumpponen A, Körner C, Leadley PW, Loreau M, Minns A, Mulder CPH, O’Donovan G, Otway SJ, Pereira JS, Prinz A, Read DJ, Scherer-Lorenzen M, Schulze ED, Siamantziouras ASD, Spehn EM, Terry AC, Troumbis AY, Woodward FI, Yachi S, Lawton JH (1999) Plant diversity and productivity experiments in European grasslands. Science, 286, 1123-1127.

Hegyi F (1974) A simulation model for managing jack-pine stands. In: Growth Models for Tree and Stand Simulation (ed. Fries J), pp. 74-90. Royal College of Forestry, Banbury.

Huang C, Sih A (1990) Experimental studies on behaviorally mediated, indirect interactions through a shared predator. Ecology, 71, 1515-1522.

Ives AR, Cardinale BJ, Snyder WE (2005) A synthesis of subdisciplines: Predator-prey interactions, and biodiversity and ecosystem functioning. Ecology Letters, 8, 102-116.

Jackrel SL, Wootton JT (2015) Cascading effects of induced terrestrial plant defences on aquatic and terrestrial ecosystem function. Proceedings of the Royal Society B:
Biological Sciences, 282, 20142522.

Johansson F (1995) Increased prey vulnerability as a result of prey-prey interactions. Hydrobiologia, 308, 131-137.

Kerr B, Riley MA, Feldman MW, Bohannan BJM (2002) Local dispersal promotes biodiversity in a real-life game of rock-paper-scissors. Nature, 418, 171-174.

Kleinhesselink AR, Kraft NJB, Levine JM (2019) Mechani sms underlying higher order interactions: From quantitat ive definitions to ecological processes. bioRxiv, https:// doi.org/10.1101/857920.

Laird RA, Schamp BS (2006) Competitive intransitivity promotes species coexistence. The American Naturalist, 168, 182-193.

Laird RA, Schamp BS (2008) Does local competition increase the coexistence of species in intransitive networks. Ecology, 89, 237-247.

Letten AD, Stouffer DB (2019) The mechanistic basis for higher-order interactions and non-additivity in competitive communities. Ecology Letters, 22, 423-436.

Levine JM, Bascompte J, Adler PB, Allesina S (2017) Beyond pairwise mechanisms of species coexistence in complex communities. Nature, 546, 56-64.

Li YZ, Mayfield M, Wang B, Xiao JL, Kral K, Janik D, Holik J, Chu CJ (2020) Beyond direct neighbourhood effects: Higher-order interactions improve modelling and predicting tree survival and growth. National Science Review, 7, https://doi.org/10.1093/nsr/nwaa244.

Liao JB, Xi XQ, Bearup D, Sun SC (2020) Metacommunity robustness of plant-fly-wasp tripartite networks with specialization to habitat loss. Ecology, 101, e03071.

Lima SL (1998) Stress and decision making under the risk of predation: Recent developments from behavioral, reproductive, and ecological perspectives. In: Advances in the Study of Behavior: Stress and Behavior (eds Moller AP, Milinski M, Slater PJB), pp. 215-290. Elsevier, Amsterdam.

Lin YC, Sutherland WJ (2014) Interaction modification effects on ecological networks are affected by ratio dependence and network topology. Journal of Theoretical Biology, 363, 151-157.

Loreau M (1998) Biodiversity and ecosystem functioning: A mechanistic model. Proceedings of the National Academy of Sciences, USA, 95, 5632-5636.

Loreau M (2000) Biodiversity and ecosystem functioning: Recent theoretical advances. Oikos, 91, 3-17.

Lorimer CG (1983) Tests of age-independent competition indices for individual trees in natural hardwood stands. Forest Ecology and Management, 6, 343-360.

Losapio G, Montesinos-Navarro A, Saiz H (2019) Perspectives for ecological networks in plant ecology. Plant Ecology \& Diversity, 12, 87-102.

Lotka AJ (1925) Elements of Physical Biology. Williams and Wilkins Company, Detroit.

Martyn TE, Stouffer DB, Godoy O, Bartomeus I, Pastore A, 
Mayfield MM (2020) Identifying 'useful' fitness models: Balancing the benefits of added complexity with realistic data requirements in models of individual plant fitness. The American Naturalist, https://doi.org/10.1086/713082.

Mayfield MM, Stouffer DB (2017) Higher-order interactions capture unexplained complexity in diverse communities. Nature Ecology \& Evolution, 1, 1-7.

Maynard DS, Crowther TW, Bradford MA (2017) Competitive network determines the direction of the diversity-function relationship. Proceedings of the National Academy of Sciences, USA, 114, 11464-11469.

Messina FJ (1981) Plant protection as a consequence of an ant-membracid mutualism: Interactions on goldenrod (Solidago sp.). Ecology, 62, 1433-1440.

Monserud RA, Sterba H (1999) Modeling individual tree mortality for Austrian forest species. Forest Ecology and Management, 113, 109-123.

Mopper S, Maschinski J, Cobb N, Whitham TG (1991) A new look at habitat structure: Consequences of herbivo re-modified plant architecture. In: Habitat Structure: The Physical Arrangement of Objects in Space (eds Bell SS, McCoy ED, Mushinsky HR), pp. 260-280. Springer Netherlands, Dordrecht.

Morin PJ, Lawler SP, Johnson EA (1988) Competition between aquatic insects and vertebrates: Interaction strength and higher order interactions. Ecology, 69, 1401-1409.

Neill WE (1974) The community matrix and interdependence of the competition coefficients. The American Naturalist, 108, 399-408.

Padilla FM, Mommer L, de Caluwe H, Smit-Tiekstra AE, Wagemaker CA, Ouborg NJ, de Kroon H (2013) Early root overproduction not triggered by nutrients decisive for competitive success belowground. PLoS ONE, 8, e55805.

Reichenbach T, Mobilia M, Frey E (2007) Mobility promotes and jeopardizes biodiversity in rock-paper-scissors games. Nature, 448, 1046-1049.

Ricker WE (1958) Handbook of Computations for Biological Statistics of Fish Populations. Fisheries Research Board of Canada, Ottawa.

Rojas-Echenique J, Allesina S (2011) Interaction rules affect species coexistence in intransitive networks. Ecology, 92, 1174-1180.

Singh P, Baruah G (2020) Higher order interactions and s pecies coexistence. Theoretical Ecology, https://doi.org/ 10.1007/s12080-020-00481-8.

Soliveres S, Lehmann A, Boch S, Altermatt F, Carrara F, Crowther TW, Delgado-Baquerizo M, Kempel A, Maynard DS, Rillig MC, Singh BK, Trivedi P, Allan E (2018) Intransitive competition is common across five major taxonomic groups and is driven by productivity, competitive rank and functional traits. Journal of Ecology, 106, 852-864.

Soliveres S, Maestre FT, Ulrich W, Manning P, Boch S, Bowker MA, Prati D, Delgado-Baquerizo M, Quero JL,
Schöning I, Gallardo A, Weisser W, Müller J, Socher SA, García-Gómez M, Ochoa V, Schulze ED, Fischer M, Allan E (2015) Intransitive competition is widespread in plant communities and maintains their species richness. Ecology Letters, 18, 790-798.

Song CL (2020) Structural stability: Concepts, methods, and applications. Biodiversity Science, 28, 1345-1361. (in Chinese with English abstract) [宋础良 (2020) 结构稳定 性: 概念、方法和应用. 生物多样性, 1345-1361.]

Spehn EM, Hector A, Joshi J, Scherer-Lorenzen M, Schmid B, Bazeley-White E, Beierkuhnlein C, Caldeira MC, Diemer M, Dimitrakopoulos PG, Finn JA, Freitas H, Giller PS, Good J, Harris R, Högberg P, Huss-Danell K, Jumpponen A, Koricheva J, Leadley PW, Loreau M, Minns A, Mulder CPH, O’Donovan G, Otway SJ, Palmborg C, Pereira JS, Pfisterer AB, Prinz A, Read DJ, Schulze ED, Siamantziouras ASD, Terry AC, Troumbis AY, Woodward FI, Yachi S, Lawton JH (2005) Ecosystem effects of biodiversity manipulations in European grasslands. Ecological Monographs, 75, 37-63.

Stouffer DB, Bascompte J (2010) Understanding food-web persistence from local to global scales. Ecology Letters, 13, 154-161.

Thébault E, Loreau M (2003) Food-web constraints on biodiversity-ecosystem functioning relationships. Proceedings of the National Academy of Sciences, USA, 100, 14949-14954.

Tilman D, Lehman CL, Thomson KT (1997) Plant diversity and ecosysterproductivity: Theoretical considerations. Proceedings of the National Academy of Sciences, USA, 94, 1857-1861.

Tscharntke $\mathrm{T}$ (1999) Insects on common reed (Phragmites australis): Community structure and the impact of herbivory on shoot growth. Aquatic Botany, 64, 399-410.

Turner A, Mittelbach G (1990) Predator avoidance and community structure: Interactions among piscivores, planktivores, and plankton. Ecology, 77, 2241-2254.

Turner AM (1996) Freshwater snails habitat use in response to predation. Animal Behaviour, 51, 747-756.

Turner AM (1997) Contrasting short-term and long-term effects of predation risk on consumer habitat use and resources. Behavioral Ecology, 8, 120-125.

Uriarte M, Condit R, Canham CD, Hubbell SP (2004) A spatially explicit model of sapling growth in a tropical forest: Does the identity of neighbours matter? Journal of Ecology, 92, 348-360.

van Veen FJF, Godfray HC (2013) Consequences of trait changes in host-parasitoid interactions in insect communities. In: Trait-Mediated Indirect Interactions: Ecological and Evolutionary Perspectives (eds Ohgushi T, Schmitz O, Holt RD), pp. 28-46. Cambridge University Press, Cambridge.

van Veen FJF, van Holland PD, Godfray HCJ (2005) Stable 
coexistence in insect communities due to density- and trait-mediated indirect effects. Ecology, 86, 3182-3189.

Vandermeer JH (1969) The competitive structure of communities: An experimental approach with protozoa. Ecology, 50, 362-371.

Verhuls PF (1838) Notice sur la loi que la population poursuit dans son accroissement. Correspondence Mathematique et Physique, 10, 113-121.

Volterra V (1926) Variazioni e fluttuazioni del numero d'individui in specie animali conviventi. Memoria della Regia Accademia Nazionale dei Lincei, 2, 31-113.

Wang SP (2020) Food web structure and functioning: Theoretical advances and outlook. Biodiversity Science, 28, 1391-1404. (in Chinese with English abstract) [王少鹏 (2020) 食物网结构与功能: 理论进展与展望. 生物多样 性, 28, 1391-1404.]

Weigelt A, Schumacher J, Walther $\mathrm{T}$, Bartelheimer $\mathrm{M}$, Steinlein T, Beyschlag W (2007) Identifying mechanisms of competition in multi-species communities. Journal of Ecology, 95, 53-64.

Werner EE, Gilliam JF (1984) The ontogenetic niche and species interactions in size-structured populations. Annual Review of Ecology and Systematics, 15, 393-425.

Werner EE, Gilliam JF, Hall DJ, Mittelbach GG (1983) An experimental test of the effects of predation risk on habitat use in fish. Ecology, 64, 1540-1548.

Werner EE, Peacor SD (2003) A review of trait-mediated indirect interactions in ecological communities. Ecology, 84, 1083-1100.

Wilbur HM (1972) Competition, predation, and the structure of the ambystoma-rana sylvatica community. Ecology, 53, 3-21.

Wootton JT (1993) Indirect effects and habitat use in an intertidal community: Interaction chains and interaction modifications. The American Naturalist, 141, 71-89.

Wootton JT (1994a) The nature and consequences of indirect effects in ecological communities. Annual Review of Ecology and Systematics, 25, 443-466.

Wootton JT (1994b) Putting the pieces together: Testing the independence of interactions among organisms. Ecology,
75, 1544-1551.

Worthen WB, Moore JL (1991) Higher-order interactions and indirect effects: A resolution using laboratory drosophila communities. The American Naturalist, 138, 1092-1104.

Wykoff WR (1990) A basal area increment model for individual conifers in the northern Rocky Mountains. Forest Science, 36, 1077-1104.

Xi XQ, Wu XW, Nylin S, Sun SC (2016) Body size response to warming: Time of the season matters in a tephritid fly. Oikos, 125, 386-394.

Xi XQ, Yang Y, Yang YH, Segoli M, Sun SC (2017) Plant-mediated resource partitioning by coexisting parasitoids. Ecology, 98, 1660-1670.

Xiao JL, Li YZ, Chu CJ, Wang YS, Meiners SJ, Stouffer DB (2020) Higher-order interactions mitigate direct negative effects on population dynamics of herbaceous plants during succession. Environmental Research Letters, 15, 074023.

Xu GH, Li XY, Shi CH (2019) The complexity-stability relationship: Progress in mathematical models. Biodiversity Science, 27, 1364-1378. (in Chinese with English abstract) [徐光华, 李小玉, 施春华 (2019) 复杂性-稳定性研究: 数学模型的进展. 生物多样性, 27, 1364-1378.]

Yang XF, Yan C, Gu HF, Zhang ZM (2020) Interspecific synchrony of seed rain shapes rodent-mediated indirect seed-seed interactions of sympatric tree species in a subtropical forest. Ecology Letters, 23, 45-54.

Yang XF, Zhang HM, Zhang ZB (2020) Mast seeding and its relationship with animal's hoarding behaviour. Biodiversity Science, 28, 821-832. (in Chinese with English abstract) [杨 锡福, 张洪茂, 张知涁 (2020) 植物大年结实及其与动物 咜食行为之间的关系. 生物多样性, 28, 821-832.]

Zhu Y, Wang DZ, Zhong ZW (2017) Characteristics, causes, and consequences of trait-mediated indirect interactions in ecosystems. Acta Ecologica Sinica, 37, 7781-7790. (in Chinese with English abstract) [朱玉, 王德利, 钟志伟 (2017) 生态系统基于性状调节的物种间接作用: 特征、 成因及后果. 生态学报, 37, 7781-7790.]

(责任编委：王少鹏 责任编辑：时意专) 